\title{
PREDICTING PERFORMANCE IN SPEED CLIMBING: ACCURACY OF THE FORCE-VELOCITY TEST ON A CYCLE ERGOMETER
}

\author{
Marcin Krawczyk \\ University of Applied Sciences in Tarnow, Poland \\ Mariusz Pociecha \\ University of Applied Sciences in Tarnow, Poland \\ Tomasz Sordyl \\ University of Applied Sciences in Tarnow, Poland \\ Aleksandra Stepek \\ University of Applied Sciences in Tarnow, Poland \\ Paulina Kozioł \\ University of Applied Sciences in Tarnow, Poland
}

\begin{abstract}
The present study examined the validity of the force-velocity (F-V) mechanical parameters of the lower limbs as a predictors of the competitive performance in junior male speed climbing. The second aim was to evaluate F-V characteristics in competitive speed climbers in the junior male category. Ten junior male speed climbers (age: $18 \pm 0.5$ years; BH: $174.7 \pm 4.5 \mathrm{~cm} ; B W: 66.4 \pm 6.6 \mathrm{~kg}$; with mean best climbing time from the competition BCT: $7.29 \pm 0.66$ s) were participants in the research. All they were participants in the Youth European Cup in speed climbing. F-V characteristics were measured using a cycle ergometer test according to the Vandewalle's protocol. To evaluate of the validity between BCT (criterion) and mechanical parameters (practical measures) from F-V test a standard error of the estimate (expressed as a raw and standardized typical error, TE) and correlation coefficients $\left(r_{x, y}\right)$ were used. Moreover, the validity of BCT as a potentially predictor of final competition rank (as a criterion for BCT) was evaluated. Additionally the mean value and standard deviation were calculated for all measured variables. Validity of the BCT as a competition place predictor was largely overestimated (TE $=0.73$ and $r_{x, y}=0.81, p p \leq 0.05$ ). Accuracy of the BCT prediction for somatic and mechanical variables were very largely error biased. The standardized values of TE for these variables were: $1.93 \div 7.25$. The $r_{x, y}$ were in the range of -0.18 to -0.46 . The level of the mechanical variables from the F-V cycle ergometer as a predictors of the BCT in speed climbing may be largely error biased. Application of the cycle ergometer F-V test of prediction BCT values in speed climbing may have low practical value. Moreover, BCT variable may be a poor predictor of the final competition result. Coaches should take these suggestions into account during preparations to speed climbing competitions.
\end{abstract}

Keywords: best climbing time, force-velocity relationship, speed climbing, sprint cycling test, validity. 
Marcin Krawczyk et al., 2021. Predicting Performance in Speed Climbing: Accuracy of the Force-Velocity Test on a Cycle Ergometer

\section{Introduction}

Power is the product of force and velocity. The power produced during muscular shortening depends on both factors. To obtain the maximal power output of the muscles the force-velocity test (F-V test) can be used. F-V test is based on relationship between frequency of revolutions and braking force and provides information about maximal velocity (V0) and maximal force (F0) capabilities. Peak power (PP) is reached at 0.5 of the F0 and V0 values. Based on this values, Vandewalle et al. (Vandewalle et al., 1987) proposed the following equation to calculate peak power: $\mathrm{PP}=0.25 * \mathrm{~F} 0 * \mathrm{~V} 0$.

In Speed Climbing duration of climbing race is approximately $6 \pm 0.23$ seconds in male and 8.05 \pm 0.36 seconds in female (Shunko \& Kravchuk, 2020). While climbing climber have to perform a lot of upper and lower limbs moves (alternately and simultaneously). The major lower limbs' function is push off the holds and to use the friction on the wall (Shunko \& Kravchuk, 2020).

Recent studies showed that the main bioenergetic source in speed climbing is the anaerobic lactate system (Krawczyk et al., 2018). Furthermore, some research showed that anaerobic power are factor which may affects the sport results in speed climbing (Krawczyk, Ozimek, et al., 2020). This established a relationship between power capabilities and speed climbing time which allows to assume that tests evaluating force, power and velocity could have practical value in predicting competition results in speed climbing.

There is only a few studies focused on force-velocity capabilities among speed climbers. Important report was made by Levernier et al. (Levernier et al., 2020). In this research a pull-ups test with load at different percentage of body mass was used to obtain upper limbs F-V profile among lead climbers, speed climbers and bouldering climbers. Another study by Krawczyk et al. (Krawczyk, Pociecha, et al., 2020) described relationships of mechanical (force, velocity and power) parameters of the countermovement jump (CMJ) with best climbing time at national championship competition.

Based on the scientific research analysis there is a gap in the literature about lower limbs F-V profile among speed climbers. The cycle-ergometer F-V test often was used in studies evaluating strength-speed capacities in similar populations of athletes (Chelly et al., 2010; Martin et al., 2003; Nikolaidis, 2012). Literature review showed no previous studies with similar objectives conducted among youth speed climbers. Therefore, one of the aim of this study was to describe F-V characteristic of the lower limbs in youth speed climbers. Moreover, above presented studies showed that the important determinants of climbing time in this discipline are force, velocity and power of the lower limbs. This means that these mechanical parameters measured in cycle-ergometer F-V test may be significantly correlated with climbing time. If so, then such test 
could be used as valuable tool in fitness testing procedures among speed climbers. Therefore, the second, and probably more important objective of this study was to establish practical validity of the mechanical parameters of the lower limbs from F-V test as a predictors of sport results at the competition in this discipline.

\section{Material and Methods}

Ten $(n=10)$ speed climbers in Junior Male category took part in this research. All climbers were participants of the IFSC European Youth Cup in speed climbing. Written informed consent was received from all participants or their guardians in case of underage climbers after verbal explanation about purposes of the study and its potential risks. All research procedures were carried out according to the Helsinki Declaration. Participants were $18 \pm 0.5$ years, with body height (BH) $174.7 \pm 4.5 \mathrm{~cm}$; body weight (BW) $66.4 \pm 6.6 \mathrm{~kg}$; best climbing time at the competition (BCT) $7.29 \pm 0.66$ (with CV $=9 \%$ ).

The F-V test was carried out using the Monark 874E according to the Vandewalle et al. (Vandewalle et al., 1987) protocol. Three maximal 6-s cycling sprinting efforts were performed with three different loads: $5 \%, 7.5 \%$ and $10 \%$ of BW.

Prior the test all participants performed a standardized warm-up procedure comprising 5 minutes of cycling. The maximal velocity values for each trial were recorded, which were used to calculate F0 and V0 values. The peak power $(\mathrm{PP})$ was calculated using the following equation: $\mathrm{PP}=0.25 * \mathrm{~F} 0 * \mathrm{~V} 0$. where $\mathrm{V0}$ - maximal theoretical revolution velocity (rev/min) and F0 - maximal theoretical braking force (kg). There was a 4 minutes rest period between each trial.

To realize the main objective of the study, mean values $(\tilde{x})$ and standard deviations (SD) for all variables were calculated. The standardized error of estimation (TE) and correlation coefficients $\left(r_{x, y}\right)$ were used to estimate the accuracy of the relationship between BCT and mechanical parameters.

Table1 Thresholds and Magnitudes for Standardized TE and Corresponding Validity of Correlations

\begin{tabular}{|c|c|c|c|}
\hline Standardized TE & Qualitativemagnitude & Validitycorrelation & Qualitativemagnitude \\
\hline$<0.1$ & trivial & $>0.995$ & excellent \\
\hline $0.1-0.3$ & small & $0.995-0.95$ & verygood \\
\hline $0.3-0.6$ & moderate & $0.95-0.85$ & good \\
\hline $0.6-1.0$ & large & $0.85-0.70$ & poor \\
\hline $1.0-2.0$ & verylarge & $0.70-0.45$ & verypoor \\
\hline$>2.0$ & impractical & $<0.45$ & impractical \\
\hline
\end{tabular}


Moreover, the accuracy of the BCT was assessed as a predictor of final position in the ranking at the competition. The calculations were made using a spreadsheets proposed by Hopkins (Hopkins, 2015).The specific threshold values for $\mathrm{TE}$ and $\mathrm{r}_{\mathrm{x}, \mathrm{y}}$ were presented in Table 1 .

\section{Results}

The obtained results of TE (0.73) and $\mathrm{r}_{\mathrm{x}, \mathrm{y}}(0.81)$ values showed that BCT as a predictor of the final competition rank could have a large error of estimate. The results presented in Table 2 showed that values of majority mechanical measured parameters and somatic features were impractical BCT estimators. Only BH, V0 and PP showed better validity, but with very poor and very large error magnitude. The results indicated in general very poor value all measured variables as a predictors of BCT.

Table 2 Values of Means, Standard Deviation, Correlation Coefficients, Typical Error of Estimate and Qualitative Magnitude for all Variables

\begin{tabular}{|c|c|c|c|c|c|c|}
\hline variables & $\tilde{\mathrm{x}}$ & $\mathrm{SD}$ & $\mathrm{r}_{\mathrm{xy}}$ & Qualitativemagnitude & $\mathrm{TE}$ & Qualitativemagnitude \\
\hline BCT [s] & 7.29 & 0.66 & & & & \\
\hline BH $[\mathrm{cm}]$ & 174.75 & 4.53 & -0.45 & verypoor & 1.98 & verylarge \\
\hline BW $[\mathrm{kg}]$ & 66.42 & 6.63 & -0.18 & impractical & 5.42 & impractical \\
\hline FM $[\%]$ & 10.61 & 3.74 & -0.14 & impractical & 7.25 & impractical \\
\hline F0 $[\mathrm{kg}]$ & 15.27 & 1.84 & -0.29 & impractical & 3.29 & impractical \\
\hline F0 $[\mathrm{kg} / \mathrm{kg}]$ & 0.23 & 0.02 & -0.26 & impractical & 3.77 & impractical \\
\hline V0 [rev/min] & 238.50 & 16.63 & -0.46 & verypoor & 1.93 & verylarge \\
\hline PP $[\mathrm{W}]$ & 913.45 & 142.73 & -0.41 & impractical & 2.2 & impractical \\
\hline PP $[\mathrm{W} / \mathrm{kg}]$ & 13.73 & 1.55 & -0.46 & verypoor & 1.94 & verylarge \\
\hline
\end{tabular}

\section{Discussion}

The main objective of this study was to evaluate the validity mechanical parameters F-V cycloergometric test as a predictors of sport results in speed climbing at Junior Male category. In general, results of this study showed low estimation accuracy of these parameters, and therefore showed potentially low practical usefulness of this type of F-V test for predicting speed climbing performance. High values of standardized error of estimation (TE) and low values of correlation coefficients $\left(\mathrm{r}_{\mathrm{x}, \mathrm{y}}\right)$ between mechanical parameters and BCT confirm this conclusion.

To date, there is a few of the research which evaluated power of the lower limbs using a cycle ergometer tests. Available data in this area is not enough basis for reliable conclusions. Krawczyk et al. (Krawczyk, Pociecha, et al., 
2020) study showed that maximal anaerobic power of Wingate Test were not significantly correlated with climbing time among youth speed climbers of both sex. Another Krawczyk et al. (Krawczyk et al., 2019) study showed significant relationship between upper limbs maximal power on Wingate Test and speed climbing performance. Based on the comparison results of present study and previous studies it is hard to state unequivocally what is the value of the practical use of cycloergometric tests in predicting the sport results in speed climbing. Out of three studies, realized in similar protocols the results of two of them showed no significant relationships between parameters from the tests and results of speed climbing competition.

These results suggested that during research among this population, jumping tests (e.g. squat jump and/or countermovement jump) could be more practical. These types of tests have already been widely used during previous research conducted among speed climbers (Krawczyk, Pociecha, et al., 2020; Krawczyk et al., 2019; Krawczyk, Ozimek et al., 2020).

It has to be mentioned, that maximal anaerobic power, strength and speed capabilities of the lower limbs are not all ingredients determining success and final results in competitive speed climbing. Other factors like technical skills, coordination abilities or mental preparedness have an impact on specific speed climbing performance. During the climbing race climbers are often making run technical errors which leads to decreasing of climbing velocity to zero or even falling of the wall. These mentioned incidents may have strongly influenced the final position in the competition ranking. Nevertheless, achieved climbing time is considered as a significant and important indicator of the specific physical performance preparedness of the speed climber and the training work effectiveness.

The recent research conducted in this area indicate differences between climbing velocity and produced kinetic energy depending on actual position-on the route during climbing (Legreneur et al., 2018, 2019). Consequently the climbing time depends on many kinetic and kinematic factors like mean climbing velocity, ability to accelerate in the spots where holds and steps configuration decreases, climber's velocity, and technical skills.

As mentioned earlier the anaerobic power is a significant factor influencing the level of movement, speed or sprint running velocity (in the case of speed climbing this will be climbing velocity). Therefore it could be hypothesized that force, velocity and power of the muscles involved during climbing significantly may determine the ability to maintain climbing velocity and rapid acceleration in proper spots. The nature of this relationships is still unknown, because there is no research describing the relationships between the results in spectrum of the non-specific tests -tests with movement similar to climbing movements kinematic and kinetic parameters observed at the route - climbing time - final 
ranking at the competition. Describing this phenomenon may enable the identification of the most important and accurate variables or tests related with sport results in speed climbing. This, in turn, may enable the assessment of the effectiveness of sport-specific training regimens and transfer results to specific speed climbing performance.

Based on this study results and recent research in speed climbing there might be a conclude that cycloergometric tests (e.g. F-V test) have poor predicting value in this specific discipline. The results of this study indicated a large errors of estimation using parameters from those type of tests. F-V tests may be a good apparatus to assess training induced changes of the strength speed performance (Buśko, 2006; Nebil et al., 2014). Furthermore, based on previous studies conducted among speed climbers it may be concluded that the jumping tests, especially in male competitors have a preferable practical value in predicting sports results in speed climbing (Krawczyk, Pociecha, et al., 2020; Krawczyk, Ozimek et al., 2020; Krawczyk et al., 2019).

\section{References}

Buśko, K. (2006). Changes of force-velocity and power-velocity relationships after training performed on cycle ergometer. Biology of Sport, 23(1), 17-32.

Chelly, M. S., Chérif, N., Amar, M. Ben, Hermassi, S., Fathloun, M., Bouhlel, E., Tabka, Z., \& Shephard, R. J. (2010). Relationships of Peak Leg Power, 1 Maximal Repetition Half Back Squat, and Leg Muscle Volume to 5-m Sprint Performance of Junior Soccer Players. Journal of Strength and Conditioning Research, 24(1), 266-271. https://doi.org/10.1519/JSC.0b013e3181c3b298

Hopkins, W. (2015). Spreadsheets for analysis of validity and reliability. Sportscience, 19, 36-42.

Krawczyk, M., Ozimek, M., Pociecha, M., Draga, P., Zadarko, E., \& Barabasz, Z. (2020). Selected morphofunctional characteristics and their correlations with performance of female and male speed climbers. Science \& Sports. https://doi.org/10.1016/ j.scispo.2019.11.009

Krawczyk, M., Ozimek, M., Rokowski, R., Pociecha, M., \& Draga, P. (2018). Anthropometric characteristics and anaerobic power of lower limbs and their raltionships with race time in female climbers. SOCIETY. INTEGRATION. EDUCATION Proceedings of the International Scientific Conference., IV, 118-126. https://doi.org/10.17770/sie2018vol1.3268

Krawczyk, M., Pociecha, M., Ozimek, M., \& Draga, P. (2020). The force, velocity, and power of lower limbs as a determinants of speed climbing efficiency. Trends in Sport Sciences, 27(4), 219-224. https://doi.org/10.23829/TSS.2020.27.4-5

Krawczyk, M, Ozimek, M., Pociecha, M., Draga, P., Zadarko, E., \& Barabasz, Z. (2020). Selected morphofunctional characteristics and their correlations with performance of female and male speed climbers. Science \& Sports, 35(4), 243-245. https://doi.org/10.1016/j.scispo.2019.11.009

Krawczyk, M, Ozimek, M., Pociecha, M., \& Gabryś, T. (2019). Morfofunkcjonalne uwarunkowania wyników sportowych we wspinaczce na czas i boulderingu. 
Wydawnictwa Państwowej Wyższej Szkoły Zawodowej w Tarnowie.

Krawczyk, M, Pociecha, M., Kozioł, P., Stepek, A., Guzy, R., Więcek, K., \& Rzepa, K. (2019). Morphological features and the strength-speed fitness of female volleyballers at a high sports level. Health Promotion \& Physical Activity, 8(3), 6-11. https://doi.org/10.5604/01.3001.0013.4330

Krawczyk, M, Pociecha, M., Ozimek, M., Stepek, A., \& Kozioł, P. (2020). Value of speed capabilities in youth speed climbing at high sports level. SOCIETY. INTEGRATION. EDUCATION. Proceedings of the International Scientific Conference, 6, 264. https://doi.org/10.17770/sie2020vol6.4862

Legreneur, P., Quaine, F., Chapelle, S., \& Reveret, L. (2018). Interpretation of hip mechanical energy in official speed climbing route. 4th IRCRA International Congress.

Legreneur, P., Rogowski, I., \& Durif, T. (2019). Kinematic analysis of the speed climbing event at the 2018 Youth Olympic Games. Computer Methods in Biomechanics and Biomedical Engineering, 22(sup1), S264-S266. https://doi.org/10.1080/ 10255842.2020.1714907

Levernier, G., Samozino, P., \& Laffaye, G. (2020). Force-Velocity-Power Profile in HighElite Boulder, Lead, and Speed Climber Competitors. International Journal of Sports Physiology and Performance, 15(7), 1012-1018. https://doi.org/10.1123/ijspp.20190437

Martin, R. J. F., Dore, E., Hautier, C. A., Van Praagh, E., \& Bedu, M. (2003). Short-Term Peak Power Changes in Adolescents of Similar Anthropometric Characteristics. Medicine \& Science in Sports \& Exercise, 35(8), 1436-1440. https://doi.org/10.1249/01.MSS.0000079074.47756.AB

Nebil, G., Zouhair, F., Hatem, B., Hamza, M., Zouhair, T., Roy, S., \& Ezdine, B. (2014). Effect of optimal cycling repeated-sprint combined with classical training on peak leg power in female soccer players. Isokinetics and Exercise Science, 22(1), 69-76. https://doi.org/10.3233/IES-130515

Nikolaidis, P. T. (2012). Age-related differences in force-velocity characteristics in youth soccer. Kinesiology, 44(2), 130-138.

Shunko, A., \& Kravchuk, T. (2020). Competitive modelling in speed climbing. BIO Web of Conferences, 26, 00051. https://doi.org/10.1051/bioconf/20202600051

Vandewalle, H., Peres, G., Heller, J., Panel, J., \& Monod, H. (1987). Force-velocity relationship and maximal power on a cycle ergometer. Correlation with the height of a vertical jump. European Journal of Applied Physiology and Occupational Physiology, 56(6), 650-656. 